\title{
Preliminary results on position reconstruction for ZEPLIN III
}

\author{
A. Lindote ${ }^{\mathrm{a}, *}$, H.M. Araujo ${ }^{\mathrm{b}}$, J. Pinto da Cunha ${ }^{\mathrm{a}}$, D.Yu. Akimov ${ }^{\mathrm{c}}$, V. Chepel ${ }^{\mathrm{a}}$, D. Davidge ${ }^{\mathrm{b}}$, \\ J.V. Dawson ${ }^{\mathrm{b}}$, M. Joshi ${ }^{\mathrm{b}}$, V.N. Lebedenko ${ }^{\mathrm{b}}$, I. Liubarsky ${ }^{\mathrm{b}}$, M.I. Lopes ${ }^{\mathrm{a}}$, F. Neves ${ }^{\mathrm{a}}$, \\ J.J. Quenby ${ }^{\mathrm{b}}$, C. Silva ${ }^{\mathrm{a}}$, V. Solovov ${ }^{\mathrm{a}}$, T.J. Sumner ${ }^{\mathrm{b}}$, C. Thorne ${ }^{\mathrm{b}}$, R.J. Walker ${ }^{\mathrm{b}}$ \\ ${ }^{a}$ LIP-Coimbra, Department of Physics, University of Coimbra, 3004-516 Coimbra, Portugal \\ ${ }^{\mathrm{b}}$ Blackett Laboratory, Imperial College, London, UK \\ ${ }^{\mathrm{c}}$ Institute of Theoretical and Experimental Physics, Moscow, Russia
}

Available online 21 November 2006

\begin{abstract}
ZEPLIN III is a two-phase xenon detector for direct dark matter search that will look for rare nuclear recoils from elastic scattering of Weakly Interacting Massive Particles off xenon atoms. ZEPLIN III measures both the scintillation and ionisation signals produced in the liquid by the interacting particle. The prompt scintillation is detected by an array of 31 photomultipliers (PM) immersed in the liquid, and a strong electric field extracts the ionisation electrons to the vapour phase. Electroluminescence photons are produced in the gas and detected by the same PM array. In this paper we describe position reconstruction methods that use both these signals to achieve a position accuracy of a few millimetres in the horizontal plane and sub-millimetre in the vertical coordinate.
\end{abstract}

(C) 2006 Elsevier B.V. All rights reserved.

PACS: 07.05.Kf; 07.05.Tp; 95.35. +d

Keywords: Detectors; Xenon; Position; Reconstruction

\section{Introduction}

ZEPLIN III is a xenon detector for direct dark matter searches soon to be deployed underground at the Boulby mine (North Yorkshire, UK). This two-phase (liquid/gas) system will look for the rare nuclear recoils (NR) that should be produced by elastic scattering of Weakly Interacting Massive Particles (WIMPs) off xenon atoms [1,2].

Neutron interactions can also cause NR and therefore constitute an irreducible background in this type of detector. This is mitigated by the use of radio-pure construction materials, by surrounding the detector with extensive hydrocarbon shielding and by deploying the system deep underground, where a large rock overburden protects it from the effects of cosmic rays. Another key challenge is to identify the very rare NR events due to WIMPs or neutrons in a background of $\beta$ and $\gamma$-rays from residual internal and external radioactivity. These electron-

\footnotetext{
${ }^{*}$ Corresponding author. Tel.: + 351239410684; fax: + 351239822358 .

E-mail address: alex@lipc.fis.uc.pt (A. Lindote).
}

recoil events are some $\sim 10^{6}$ times more abundant and must be effectively discriminated from NR.

ZEPLIN III measures both the scintillation and ionisation produced in liquid xenon by the interacting particle. The scintillation is measured by 31 photomultipliers (PMT) immersed in the liquid. A strong electric field removes ionisation from the interaction point and extracts it into the gas. Electroluminescence photons are then produced and detected by the same PMT array. A sensitivity down to one electron extracted from the liquid is anticipated.

In order to achieve the excellent discrimination ability required to fully separate the electron and NR populations, a very uniform response is necessary for both channels across the active volume. In most detectors, this fiducialisation is achieved by physically delimiting the active region. In ZEPLIN III, this is accomplished by reconstructing the three-dimensional interaction point and rejecting events occurring in out-lying regions, where light collection and electric field may not be uniform. Therefore, position reconstruction is key in determining the ultimate sensitivity to WIMPs. 


\section{The ZEPLIN III detector}

ZEPLIN III consists of an inner vessel containing the xenon target and a liquid $\mathrm{N}_{2}$ reservoir, which provides cooling of the target to $-100^{\circ} \mathrm{C}$. These are located inside a larger vessel for vacuum insulation. It is built mostly from high-purity copper, which keeps the radioactive backgrounds to a minimum.

Inside the target chamber, a hexagonal array of 31 PMTs (2-in. diameter, $\sim 30 \%$ quantum efficiency [2]) is placed below the 40-mm high xenon volume, immersed in the liquid to enhance light collection, in particular that from primary scintillation. This is crucial given the very small number of photoelectrons expected from WIMP interactions. This geometry leads to an expected light yield of $\sim 3.5$ photoelectron ( $\mathrm{phe}$ ) $/ \mathrm{keV}$ (with no electric field) for $\gamma$-ray interactions $[2,3]$.

A strong electric field (up to $8 \mathrm{kV} / \mathrm{cm}$ in liquid, $16 \mathrm{kV} / \mathrm{cm}$ in gas) is applied between the top mirror and a wire grid $5 \mathrm{~mm}$ above the PMTs to extract the ionisation from the track into the 5-mm thick gas layer which exists above the liquid. The density of this ionisation is lower for electron interactions than for NR, making charge collection from these tracks easier. Electrons that do not recombine are then drifted upwards and extracted into the gas, where they acquire enough energy to produce excitation and electroluminescence in gaseous xenon. Operating at the maximum field one single electron can produce as many as $500 \mathrm{VUV}$ photons, of which an average $\sim 26$ are detected as phe in the PMTs [3].

These two signals provide both discrimination of the type of interacting particle and spatial information concerning the interaction point. The ratio between the scintillation $\left(S_{1}\right)$ and the electroluminescence signals $\left(S_{2}\right)$ is higher for $\gamma$-rays, allowing a rejection fraction as good as $10^{-5}$ while maintaining a good detection efficiency for NR down to a few $S_{1}$ phe [4]. This result points to a threshold of $\sim 2 \mathrm{keV}$ in zero-field visible energy $(\sim 10 \mathrm{keV}$ recoil).

\section{Position reconstruction}

Three-dimensional information on each event will allow the identification of peripheral interactions and the fiducialisation of the active volume. Particles interacting in outlying regions, where the electric field is not uniform, can therefore be identified. Small-energy deposits from $\alpha$ particles and NR arising from radioactive contamination of the detector surfaces can also be excluded.

The $z$ coordinate can be calculated from the time difference between $S_{1}$ and $S_{2}$, using the saturation drift velocity in liquid xenon $(\sim 3 \mathrm{~mm} / \mu$ s at high field [5]), with an accuracy of $\sim 0.1 \mathrm{~mm}$.

\section{1. $x-y$ reconstruction}

Previous work on $x-y$ position reconstruction in ZEPLIN III included an analytical approach [6] and a simulation-based template method [7]. Here we develop the latter approach further.

A full Monte Carlo simulation of the detector [3] was used to create a map of light collection for $\sim 20000$ points in the gas (only the $S_{2}$ signal is needed for the $x-y$ position reconstruction), organized in a $2.5-\mathrm{mm}$ square mesh. For each of these points $\sim 1.5 \times 10^{6}$ VUV photons were generated and propagated inside the detector, and the resulting signals in each PMT stored. The same simulation was then used to calculate the detector response for 1,4 and 10 electrons extracted from the liquid, from randomly generated points inside the target volume.

For each of these sample points a $\chi^{2}$ comparison is performed sequentially for each template point; the one with the lowest $\chi^{2}$ value being selected and retrieved as the best estimate position.

This simple method provides good resolution results, but the search process can be a lengthy one. A requirement of the reconstruction algorithm is that it is able to run on-line during data acquisition ( $\sim 5$ events/s when deployed underground). In order to ensure the required computational performance of the algorithm, the full search was replaced by a sampled search, in which only a fraction of the template nodes are tested. For this purpose a 2-dimensional Sobol sequence [8] was used, as it provides a more uniform spatial coverage than a pure random one (as shown in Fig. 1). The template can now be searched only in the points retrieved from this sequence, allowing a much shorter processing time. In order to maintain the reconstruction accuracy a second search is then performed in the neighbourhood of the selected point.

We decided for the use of 2500 points in the Sobol sequence, as it was observed that reconstruction quality was not affected beyond this limit. Using this method the algorithm was now able to perform at $\sim 200 \mathrm{~Hz}$ in a $3 \mathrm{GHz}$ CPU-nearly 10 times faster than the complete search version.

\section{Results}

Fig. 2 shows the reconstructed coordinates as a function of the real coordinates for 1 and 10 extracted electrons. We see that, even if only one electron is extracted, reconstruction is possible up to the limits of the PMT array $(R \sim 160 \mathrm{~mm})$. As expected, the quality of the reconstruction is significantly better for the case of 10 electrons.

Table 1 summarizes the achieved resolutions (FWHM, in $\mathrm{mm}$ ) obtained by Gaussian fits for the 3 samples in different regions of the detector: near the centre $(R<135 \mathrm{~mm})$, up to the margins of the PMT array $(R<155 \mathrm{~mm})$, and in the most outward part of the latter $(135 \mathrm{~mm}<R<155 \mathrm{~mm})$, where the light collection is lower and less uniform. It is clear that a resolution of $\sim 2 \mathrm{~cm}$ is possible even for the worst possible case of only 1 extracted electron in the outskirts of the PMT array. 

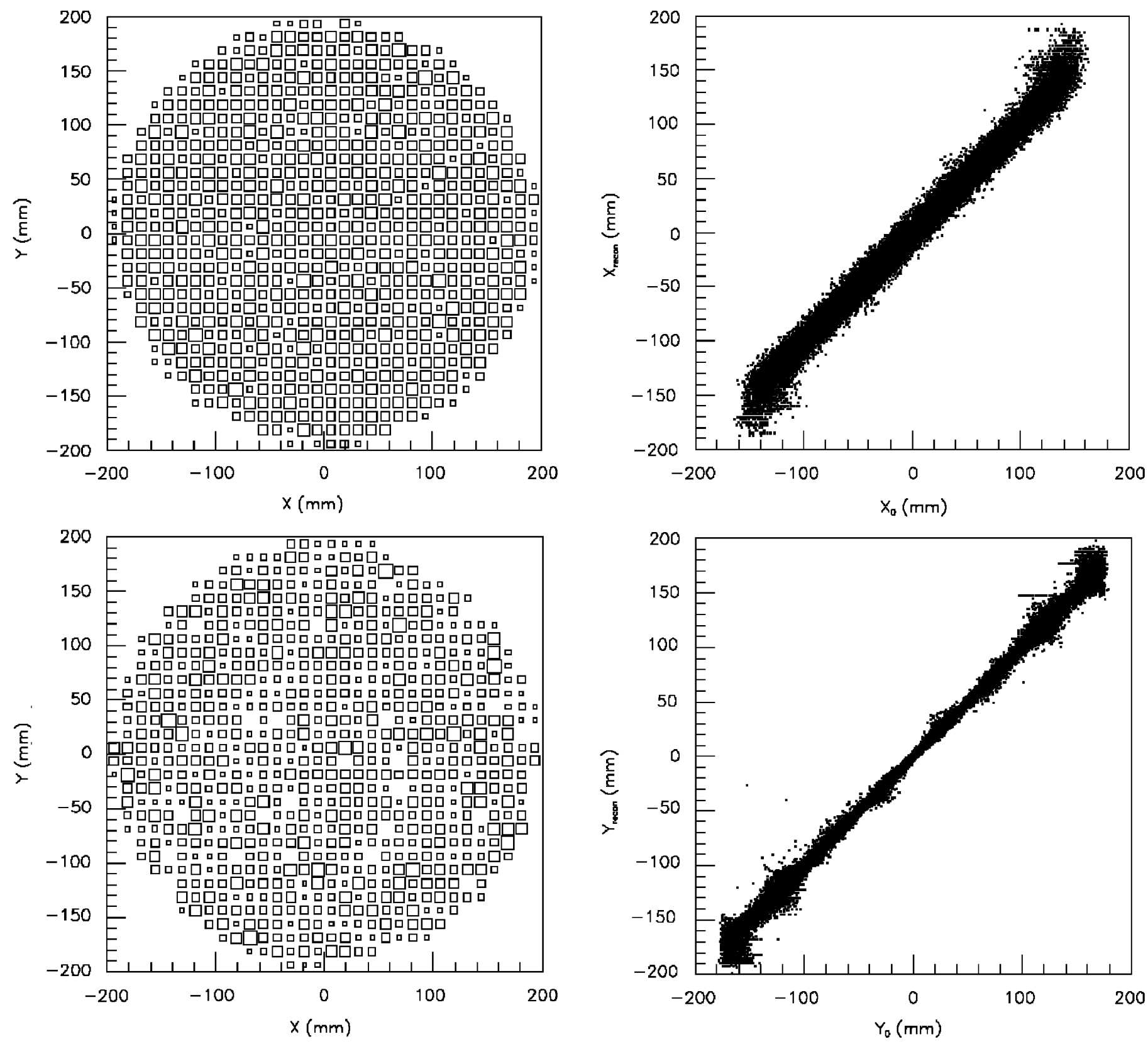

Fig. 1. A 2-D Sobol distribution (top) provides a much better spatial uniformity than a pure random one (bottom). The size of each square reflects the local point density.

With a $\sim 10 \mathrm{keV}$ NR determined by $S_{1}$, we expect an $S_{2}$ signal above 10 extracted electrons [9], and thus a resolution better than a few $\mathrm{mm}$.

An important information derived from the reconstruction results is the boundary of the fiducial volume (defined as the volume where each event can be fully characterized). The fiducial xenon mass will ultimately determine the dark matter limit of the detector. The reconstruction fails clearly for events occurring above a radius of $160 \mathrm{~mm}$, which coincides both with the boundaries of the PMT array and with the region where the electric field uniformity begins to break. Table 2 shows the contamination (i.e., out-lying events wrongly repositioned inside the ad hoc fiducial

Fig. 2. Reconstructed coordinate versus real coordinate for 1 (top) and 10 (bottom) extracted electrons.

volume) for several volumes and different cuts in the $S_{2}$ amplitude, for 10 electrons extracted from the liquid. We see that the contamination is quite small for all the tested volumes and amplitudes.

\section{Conclusions}

We have implemented a position reconstruction method for the ZEPLIN III detector. It is fast enough for on-line operation and provides a resolution of a few millimetres (FWHM) at the $S_{1}$ energy threshold, when $\sim 10$ electrons are extracted from the liquid phase (and better than $2 \mathrm{~cm}$ if only one electron is extracted). A fiducial volume up to $8 \mathrm{~kg}$ 
Table 1

Resolutions (in mm, FWHM) for the reconstruction of the 3 samples, in 3 regions of the xenon target volume

\begin{tabular}{lcccc}
\hline Extracted electrons & $\left\langle S_{2}\right\rangle$ phe & $R<135 \mathrm{~mm}$ & $R<155 \mathrm{~mm}$ & $135 \mathrm{~mm}<R<155 \mathrm{~mm}$ \\
\hline 1 & 25 & 16.6 & 17.2 & 19.9 \\
4 & 100 & 8.4 & 9.2 & 12.5 \\
10 & 250 & 4.9 & 5.5 & 8 \\
\hline
\end{tabular}

Table 2

Relative contribution (in \%) of out-lying events $(R>160 \mathrm{~mm})$ to the total number of events reconstructed inside several fiducial volumes for 10 electrons extracted and 3 different cuts in $S_{2}$

\begin{tabular}{rlccc}
\hline$S_{2} \geqslant$ (phe) & \multicolumn{4}{l}{ Xenon volume $(\mathrm{kg})$} \\
\cline { 2 - 5 } & 6 & 7 & 7.5 & 8 \\
\hline 25 & 0.0 & 0.1 & 0.4 & 1.6 \\
50 & 0.0 & 0.1 & 0.2 & 1.1 \\
100 & 0.0 & 0.0 & 0.0 & 0.4 \\
\hline
\end{tabular}

is attainable with low contamination from out-lying events and good reconstruction accuracy.

\section{Acknowledgement}

A. Lindote acknowledges the support from Fundação para a Ciência e Tecnologia, Portugal.

\section{References}

[1] T.J. Sumner, in: N.J.C. Spooner, V. Kudryavtsev (Eds.), Proceedings of the Third International Workshop on the Identification of Dark Matter, World Scientific, Singapore, 2001, p. 452.

[2] H.M. Araújo, et al., in: Proceedings of the 2005 IEEE International Conference on Dielectric Liquids, Coimbra, Portugal, 2005, pp. 305-308.

[3] H.M. Araújo, ZepIII: A (GEANT4) Simulation Tool for ZEPLIN III, Internal Report, Imperial College London, 2001.

[4] J. Dawson, Ph.D. Thesis, Imperial College London, University of London, 2003.

[5] L.S. Miller, et al., Phys. Rev. 166 (3) (1968) 871.

[6] V.A. Ditlov, et al., in: N.J.C. Spooner, V. Kudryavtsev (Eds.), Proceedings of the Fourth International Workshop on the Identification of Dark Matter, World Scientific, Singapore, 2002, pp. 383-389.

[7] D. Davidge, Ph.D. Thesis, Imperial College London, University of London, 2004.

[8] W.H. Press, et al., Numerical Recipes in C, second ed., Cambrigde University Press, New York, 1994.

[9] E. Aprile, et al., in: Proceedings of the 2005 IEEE International Conference on Dielectric Liquids, Coimbra, Portugal, 2005, pp. 313-316. 